\title{
Miranda
}

Revue pluridisciplinaire du monde anglophone /

Multidisciplinary peer-reviewed journal on the English-

speaking world

1 | 2010

Variations on Darwin

\section{Genre et héritage darwinien chez les poétesses victoriennes}

Fabienne Moine

(2) OpenEdition

Journals

Édition électronique

URL : http://journals.openedition.org/miranda/573

DOI : $10.4000 /$ miranda. 573

ISSN : 2108-6559

Éditeur

Université Toulouse - Jean Jaurès

\section{Référence électronique}

Fabienne Moine, «Genre et héritage darwinien chez les poétesses victoriennes », Miranda [En ligne], 1। 2010, mis en ligne le 23 mars 2010, consulté le 16 février 2021. URL : http://journals.openedition.org/ miranda/573 ; DOI : https://doi.org/10.4000/miranda.573

Ce document a été généré automatiquement le 16 février 2021.

\section{(c) $(1) \Theta \theta$}

Miranda is licensed under a Creative Commons Attribution-NonCommercial-NoDerivatives 4.0 International License. 


\title{
Genre et héritage darwinien chez les poétesses victoriennes
}

\author{
Fabienne Moine
}

\begin{abstract}
V'là trois millions d'années que j'dormais dans la tourbe Quand un méchant coup d'pioche me trancha net le col Et me fit effectuer une gracieuse courbe A la fin de laquelle je plongeai dans l'formol D'abord on a voulu m'consolider la face On se mit à m'brosser mâchoire et temporal Suivit un shampoing au bichromat' de potasse Puis on noua un' faveur autour d'mon pariétal. Ils ont dit que j'vivais jadis dans une grotte Ils ont dit tell'ment d'choses tell'ment de trucs curieux Qu'j'étais couvert de poils et qu'j'avais pas de culotte Alors que j'habitais un pavillon d'banlieue J'étais comm' tout le mond' pétri de bonn's manières Tous les dimanch' matins je jouais au tiercé Je portais des cols durs et des bandag's herniaires.
\end{abstract}

1 Dans L'Homme fossile, chanson très populaire de 1968, son interprète, Serge Reggiani, se moque des paléontologues qui vouent un véritable culte à ce squelette de pithécanthrope. Si, il y a trois millions d'années, cet hominidé jouait au tiercé et vivait en banlieue, c'est que l'être humain moderne a bien peu évolué ! Humour, chanson et théorie de l'évolution : trois éléments qui s'associent parfaitement lorsqu'il s'agit de se moquer de l'époque dans laquelle on vit et de nos frères humains qui semblent être restés à l'âge de pierre! Cent ans plus tôt, The Descent of Man, and Selection in Relation to Sex allait déclencher les critiques, les sarcasmes et parfois les rires des compatriotes de Darwin. C'est après la publication de The Descent of Man en 1871 et non après celle de On the Origin of Species en 1859 que les poétesses de la fin du XIX ${ }^{\mathrm{e}}$ siècle vont prendre également part aux débats soulevés par les théories sur l'évolution et sur la sélection naturelle et sexuelle. En parallèle avec le mouvement de revendication des droits de la femme qui s'intensifie à partir des années 1880, certaines poétesses victoriennes choisissent une poésie à coloration scientifique pour répondre aux théories darwiniennes appliquées à l'espèce humaine. L'appropriation et parfois la confusion 
entre Darwinisme, Lamarckisme et évolutionnisme social conduisent ces poétesses à proposer un discours sous forme poétique sur la question des rapports de sexe. Mais la réponse poétique au Darwinisme se fait toujours à travers une poésie qui adopte les codes du discours scientifique. Appropriation du discours généralement réservé aux hommes, utilisation ironique des codes, féminisation du genre de la littérature scientifique, tels sont les usages que plusieurs femmes vont faire dans leur poésie qui articule héritage darwinien et questions de genre. On pourrait facilement être surpris par une telle production littéraire lorsqu'on se souvient du fossé qui sépare la science de la poésie selon Wordsworth dans la Préface à Lyrical Ballads (1802), puis chez les penseurs victoriens qui considèrent que l'objectivité de la science est incompatible avec la subjectivité de la poésie. Mais les avancées scientifiques et médicales n'indiquentelles pas que la science est aussi idéaliste ? De même, la poésie victorienne n'a-t-elle pas des échos positivistes et progressistes lorsqu'elle se mêle de dénoncer les maux de la société, et dans notre cas précis, l'ampleur du fossé social entre les sexes! C'est précisément la question du genre qui établit un lien entre science et poésie et autorise la femme artiste à se trouver une place homologuée au cœur de ces deux approches. L'investissement des poétesses victoriennes dans le champ scientifique ne peut être dissocié d'une prise de position personnelle - et souvent féminine - face aux implications sociales que peuvent avoir les théories darwiniennes. Provoquant tantôt l'enthousiasme, tantôt l'amertume des propos féministes, ces théories sont illustrées dans une poésie aux visages multiples puisqu'elle réussit à combiner vulgarisation du discours scientifique contemporain, perspectives sociales et ambition esthétique. A la critique sociale, présente chez Mathilde Blind, Louisa Bevington et A. Mary F. Robinson, s'ajoute l'outil humoristique qui donne à leurs poèmes un pouvoir atemporel, au-delà des variations que les mœurs peuvent subir sous l'effet du temps. Constance Naden d'abord, puis May Kendall, pour achever ces fouilles poétiques, sont deux poétesses de la fin du XIX $\mathrm{X}^{\mathrm{e}}$ siècle qui choisissent l'ironie, l'humour et la dérision, plutôt que l'écœurement, le mépris et le sarcasme, pour exprimer leur opinion sur l'essentialisme ou l'individualisme que la lecture des œuvres de Darwin peut parfois soulever.

2 La science et la question de la femme sont très souvent liées chez les Victoriens. Si ce qu'on appelle gendered science, c'est-à-dire la science qui porte sur l'étude des disparités entre les sexes, n'est pas une approche exceptionnelle, ce n'est que dans les deux dernières décennies du XIX ${ }^{e}$ siècle que les textes théoriques et critiques les plus riches furent publiés à ce sujet. Et à l'époque où Charles Darwin publie The Descent of Man, la théorie scientifique ne manque souvent pas d'apporter les éléments qui confortent l'idéologie des sexes. Certes, le texte darwinien a permis d'encourager les progrès sociaux permettant une plus grande autonomie des femmes, mais il a aussi contribué à confirmer la hiérarchie entre les sexes que le discours idéologique victorien construit depuis une cinquantaine d'années. Darwin soutient que les capacités intellectuelles des hommes sont supérieures à celles des femmes. Celles-ci ont une plus grande capacité d'intuition, de perception et d'imitation qui les rapprochent des êtres moins évolués. $\mathrm{Au}$ contraire, les hommes ont des capacités intellectuelles supérieures qui leur permettent de réussir avec un plus grand succès, "a higher eminence ", ce qu'ils entreprennent (Darwin 381). Cependant, Darwin considère que les femmes possèdent des qualités maternelles qui sont à la base de l'instinct social permettant au scientifique de placer en elles ses espoirs de conduire l'évolution, éthique cette fois-ci, de l'humanité. Mais cette remarque sert aussi à confirmer les théories essentialistes qui établissent une hiérarchie des sexes et qui leur attribuent des qualités différentes. 
Toujours selon Darwin qui reprend les remarques de Francis Galton sur le génie et l'hérédité, si l'on établissait deux listes des hommes et des femmes les plus doués dans le domaine des arts, des sciences humaines et des sciences de la nature, celle des esprits éclairés masculins serait bien plus longue.

3 Ainsi, pour infirmer l'idée que la science et la poésie sont des domaines où excellent les hommes grâce à leur supériorité intellectuelle et pour montrer que ce discours idéologique est sujet à controverses multiples, il suffit de considérer quelques poétesses victoriennes qui prennent en charge le discours scientifique traditionnellement réservé aux hommes et expriment avec succès, et souvent à travers le mot d'esprit - encore un domaine que l'on croit réservé aux hommes - des positions intellectuelles fortes. Ce que les poétesses ajoutent dans leurs commentaires poético-scientifiques, c'est l'espoir qu'il existe une analogie entre le naturel et le social qui conduira à une amélioration de l'espèce humaine. C'est précisément cette question de l'évolution sociale qu'elles pointent dans leurs poèmes, parfois pour la soutenir, parfois pour la rejeter. De la même manière que la poésie féminine est un outil privilégié de revendication sociale et fonctionne comme instrument d'homologation de l'entrée des femmes dans le monde privilégié des arts, la poésie scientifique que choisissent certaines d'entre elles apporte la sanction que le genre féminin peut doublement contribuer à la dissémination des idées et des arts. Mais on ne saurait lire cette poésie de femmes comme un rejet total de l'idéologie des sexes puisque leur poésie contribue aussi à la construction des disparités essentialistes. Les émotions dont sont supposées faire preuve les femmes, et qui plus est les poétesses, sont aussi un argument qu'elles mettent en avant dans une poésie positiviste qui conduit le genre humain sur la route de la perfection. La poésie scientifique de femmes se présente donc comme une arme à double tranchant qui dénonce la question de la hiérarchie des sexes mais qui confirme par ailleurs les spécificités de l'âme et donc de l'écriture des femmes poètes.

Mathilde Blind fait partie des enthousiastes des théories darwiniennes. Elle consacre tout un volume de poèmes à la science en y louant ses objectifs progressistes. Trois longs poèmes composent The Ascent of Man (1889) qui ne cache pas sa filiation darwinienne. La question de la filiation qui est au cœur du livre de Darwin est précisément l'objet des poèmes scientifiques de Blind qui conçoit l'évolution des espèces jusqu'à l'homme comme un progrès, une "ascension ", une marche en avant, d'où le choix de « ascent » qui met en évidence cette notion d'amélioration absente du titre darwinien. "Chaunts of Life", première partie de The Ascent of Man, est une épopée évolutionniste. Je ne considérerai que ce poème car il fait, plus que les autres, directement appel aux théories darwiniennes. Mathilde Blind y décrit d'abord l'évolution depuis la naissance de l'univers jusqu'aux premiers frémissements de la vie. C'est l'amour, principe féminin et maternel selon elle, qui insuffle la vie sur terre :

Lo, moving o'er chaotic waters,

Love dawned upon the seething waste,

Transformed in ever new avatars

It moved without or pause or haste:

Like sap that moulds the leaves of May

It wrought within the ductile clay.

And vaguely in the pregnant deep,

Clasped by the glowing arms of light

From an eternity of sleep

Within unfathomed gulfs of night

A pulse stirred in the plastic slime 
Responsive to the rhythm of Time. (Blind « Chaunts of Life », 8)

5 Mais une fois que les espèces veulent se propager et évoluer, l'amour est indissociable de la luxure (« lust of life »), à laquelle Blind donne un visage masculin :

The lust of life's delirious fires

Burned like a fever in their blood,

Now pricked them on with fierce desires,

Now drove them famishing for food,

To seize coy females in the fray,

Or hotly hunted for prey. (Blind 11)

Ainsi commence la longue lutte pour la vie :

War rages on the teeming earth;

The hot and sanguinary fight

Begins with each new creature's birth:

A dreadful war where might is right;

Where still the strongest slay and win,

Where weakness is the only sin. (Blind 12)

7 Puis apparaît le fragile humain qui devient peu à peu l'être évolué capable de maîtriser le feu :

From age to dumb unnumbered age,

By dim gradations long and slow,

He reaches on from stage to stage,

Through fear and famine, weal and woe

And, compassed round with danger, still

Prolongs his life by craft and skill.

With cunning hand he shapes the flint,

He carves the horn with strange device,

He splits the rebel block by dint

of effort - till one day there flies

A spark of fire from out the stone:

Fire which shall make the world his own. (Blind 15-16)

Le monde qu'il construit est fait de violence, de vols et de meurtres. Inspiré par une force de vie, l'homme développe ses qualités intellectuelles et son imagination, ce qui se caractérise dans le poème par un changement poétique formel. Alors que la longue avancée de la vie jusqu'à l'homme moderne qui construit des villes est transcrite sous la forme régulière du pentamètre iambique, les vers longs et courts de la seconde partie de «Chaunts of Life » représentent, à la manière des calligrammes, la construction et la destruction des villes et des empires. Le poème se poursuit par la succession des religions polythéistes, puis le Christianisme rédempteur, immédiatement mis à mal par les atrocités des églises. Le monde semble être au bord du chaos à cause de la force destructrice de l'homme. Seul le poète peut favoriser la communication entre les êtres, reconstruire un monde qui se fonde autour des principes de l'amour et de la paix. Il reconnaît une harmonie naturelle et un rythme dans le monde derrière le désordre apparent causé par la cupidité et la violence des désirs humains :

Who can unseal the heart with all its tears,

Marshal loves, hates, hopes, sorrows, joys, and fears

In quick procession o'er the passive pages;

Who has given tongue to silent generations

And wings to thought. [...]

The poet, in whose shaping brain

Life is created o'er again

With loftier raptures, loftier pain; 
Whose mighty potencies of verse

Move through the plastic Universe,

And fashion to their strenuous will

The world that is creating still. (Blind 54)

La poésie permet une transcendance des disparités sexuelles, nationales, religieuses, autorisant la perception chrétienne d'une universalité et d'une atemporalité fondée sur l'harmonie et l'amour. L'homme et la nature sont finalement unis dans un principe universel et mystique. Fervente darwiniste, Blind voulait ajouter à l'aridité du discours scientifique sa conviction qu'il existe une compassion universelle, ce que The Descent of Man défendait en élaborant la théorie que les instincts sociaux et le sentiment de sympathie entre hommes compensent l'œuvre de la sélection naturelle. Darwin voit dans cette propension à soutenir son prochain l'expression de la moralité et de la rationalité ; Blind y ajoute sa marque de poète mystique qui voit dans le poète celui qui contribue à un développement chrétien de l'âme humaine que l'homme a contribué à corrompre. Pour conclure sur la présentation de cette première poétesse, je voudrais signaler que la deuxième édition de 1889 de The Ascent of Man fut publiée avec une introduction d'Alfred Russel Wallace, scientifique qui était arrivé à des conclusions similaires à celles de Darwin et qui accéléra la publication des recherches de ce dernier et dont Blind se fait l'écho dans son poèmes. Une force supérieure, certes non chrétienne, est à l'œuvre dans la nature, guidant l'homme dans son développement. Pour Blind, cette force supérieure qui s'appelle l'amour peut être appréhendée et diffusée par le poète. Ainsi la fusion de la poésie et de la science est totale dans cette épopée scientifique et mystique.

La combinaison de la science et de l'éthique doit conduire au progrès social auquel les poétesses victoriennes sont très attachées. Si Blind choisit l'épopée, genre masculin, pour donner sa version de l'évolution, d'autres poétesses choisissent d'autres modalités plus courtes et plus incisives lorsqu'il s'agit d'opposer l'évolution physique et intellectuelle de l'espèce au développement inverse de la moralité. Un détour par l'anarchiste Louisa Bevington et par la passionnée d'archéologie A. Mary F. Robinson s'impose lorsqu'il s'agit de considérer que les théories évolutionnistes trouvent un écho défavorable dans la poésie engagée qui s'attache à décrire les résultats d'une évolution compromise lorsque l'homme atteint un certain niveau de civilisation. Louisa Bevington (1845-1895) est une anarchiste qui propose dans sa poésie et sa prose une vision politique progressiste. Elle utilise le discours darwinien compatible avec son idéologie sociale lorsqu'elle dénonce l'égocentrisme des hommes qui conduit à l'oppression des peuples. Deux petits poèmes évoquent les théories scientifiques en vogue : « Egoisme à Deux » (1882) présente une description de la création du monde en termes géologiques et évolutionnistes. Ces théories sont suivies de la possibilité qu'une force supérieure, une divinité, commanderait les forces naturelles afin de conduire deux êtres à se rencontrer et à s'aimer. La question rhétorique finale "Was it divine? » souligne que la rencontre est accidentelle tout comme notre existence et la vie en général sur la planète. Bevington, radicale, athée et disciple de Herbert Spencer, rappelle que l'expérience des hommes est le fruit du hasard. Celui qui croit qu'il est unique devra alors apprendre à modérer ses ardeurs. C'est l'objet du poème « Measurements » qui met en parallèle la petitesse de notre planète et du genre humain avec la grandeur de l'amour qui fait de l'instant une éternité. Tout est relatif et question de perception. Alors relativisons un peu les expériences que nous croyons extraordinaires. Les théories scientifiques et darwiniennes entre autres sont utilisées 
ici pour montrer que la perception humaine est réduite et fausse par rapport à l'étendue du pouvoir de la nature qui poursuit une véritable évolution et que l'homme cherche à maîtriser par égocentrisme et par anthropomorphisme.

11 La poétesse A. Mary F. Robinson adapte le monde qui l'entoure, scientifique ou artistique, afin de livrer un message philosophique. Dans son poème "Darwinism " (1888), elle emprunte l'évolution et son discours pour montrer que les êtres évoluent parce qu'ils ne sont pas heureux dans l'état où ils se trouvent. La fougère qui ne peut pas fleurir parvient à force de volonté à produire une fleur. La fleur devient fruit croqué par le sémillant chimpanzé qui commence alors à sentir en lui une détresse lointaine, la même que ressentaient déjà les autres êtres vivants. L'angoisse est toujours présente chez l'homme qui attend la nouvelle forme d'évolution comme la solution à son inquiétude existentielle :

And now the same unrest

Goads to the same invisible goal,

Till some new gift, undream'd, unguess'd,

End the new travail of the soul. (A. Mary F. Robinson, "Darwinism », Leighton et

Reynolds, 547)

Chaque strophe comporte plusieurs adjectifs ou noms précédés du préfixe privatif « un» qui signale que tout est encore à faire (« unflowering; unconscious; unrest; unblossoming; unquiet; unchanged; unhappiness ; unborn ; unrest ; undream'd ; unguess'd»). Le mot porte en gestation l'idée qu'il cherche à produire, mais le préfixe rejette la réalisation dans un autre temps. Évolution et désarroi sont indissociables chez Robinson, qui remet en cause le terme même d'évolution. Elle lui préfère celui de « Darwinisme » dans le titre pour mettre en évidence l'ironie du progrès fondé sur le sentiment d'incomplétude.

13 L'ironie qui fait la force du poème social chez Bevington et du poème existentiel chez Robinson devient l'outil privilégié des poétesses fin-de-siècle qui trouvent dans le trait d'esprit et l'humour la distance qui favorise le jugement personnel et la subjectivité tandis que la science fait une démonstration objective, prétendument masculine, ne laissant pas de place au doute ni à l'éthique. Constance Naden incarne parfaitement les liens et tensions qui peuvent exister entre science et éthique puisque c'est une poétesse qui a étudié les sciences. Elle est influencée comme beaucoup d'autres femmes par la pensée de Herbert Spencer et sa philosophie scientifique, ce qui la conduit à considérer les théories darwiniennes avec une certaine distance critique qu'elle exprime dans certains poèmes drôles et ironiques. "Evolutionary Erotics » (1887), poème en quatre tableaux, utilise le discours évolutionniste pour décrire les relations amoureuses. L'ensemble des théories darwiniennes, évolution, sélection naturelle et sexuelle, se retrouvent dans la forme et le contenu de ces poèmes. "Scientific Wooing " est un poème travesti, c'est-à-dire un monologue écrit par une femme et dont le narrateur est un homme. La remise en cause de l'idéologie qui entoure les sexes est incontournable et s'effectue au moyen du discours scientifique androcentré. Le locuteur raconte d'abord qu'il était un jeune homme passionné de science :

I was a youth of studious mind,

Fair Science was my mistress kind,

And held me with attraction chemic;

No germs of Love attacked my heart,

Secured as by Pasteurian art

Against that fatal epidemic.

For when my daily task was o'er 


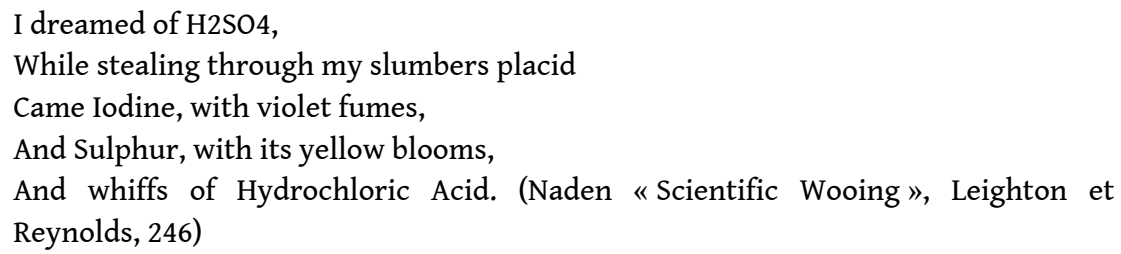

Malheureusement, un jour, il tombe amoureux de Mary Maud Trevylyan qui l'obsède : son image s'incruste dans les cellules de son cerveau. Il cherche alors à élaborer des stratégies pour la séduire qui ne sont que la réactualisation scientifique du jeu amoureux traditionnel qui transforme l'amante en objet de vénération et ici d'observation scientifique. En témoigne l'intégration du discours fleuri et du discours scientifique jusqu'à l'amalgame total :

I'll sing a deep Darwinian lay

of little birds with plumage gay,

Who solved by courtship Life's enigma;

I'll teach her how the wild-flowers love,

And why the trembling stamens move,

And how the anthers kiss the stigma.

Or Mathematically true

With rigorous Logic will I woo,

And not a word I'll say at random;

Till urged by Syllogistic stress,

She falter forth a tearful "Yes, »

A sweet «Quod erat demonstrandum! » (Naden 248-249)

15 Au lieu de s'adresser aux dieux de l'amour, il invoque ceux de la chimie, Robert Boyle, Amadeo Avogadro et Humphry Davy à son secours. Dans le deuxième tableau, «The New Orthodoxy", la critique s'adresse aux dames cette fois-ci puisque le poème reproduit la forme d'une lettre d'Amy qui quitte son amant parce qu'il a perdu la foi en Spencer et en Darwin. Fred voulait faire d'elle une femme cultivée, ce qu'elle est devenue. Elle peut lors utiliser des arguments masculins pour lui exprimer sa déception :

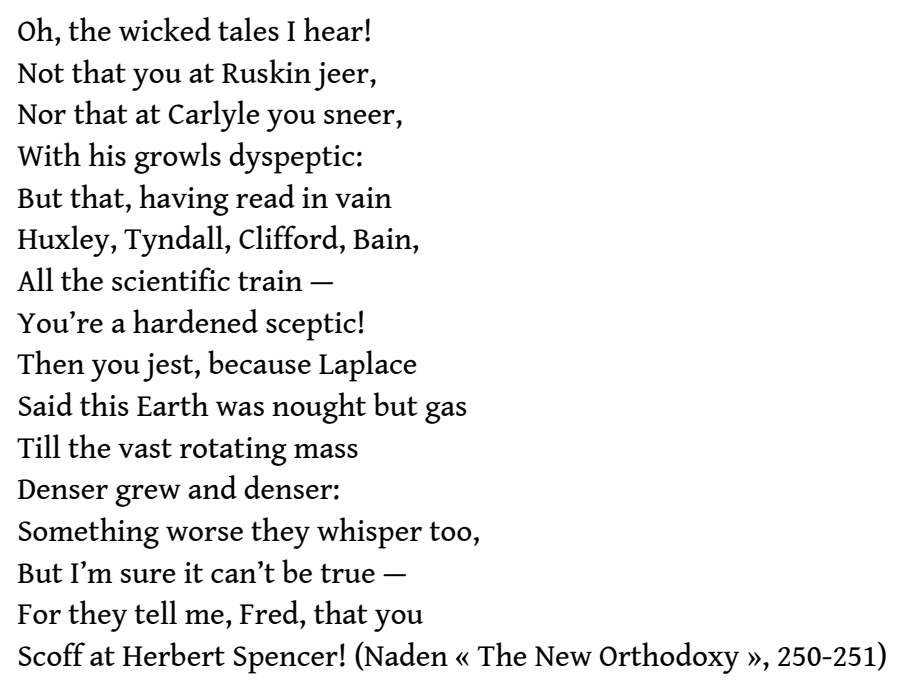

Les arguments déployés sonnent faux dans la bouche de celle qui les emprunte, car le schéma amoureux n'est pas inversé, ne donnant ni autorité ni autonomie à la locutrice. Elle confirme ce que Darwin explique lorsqu'il insiste sur les facultés d'imitation des femmes. Ici ses connaissances acquises font d'Amy le véhicule irréfléchi de théories scientifiques qu'elle répète naïvement. "Natural Selection » rapporte les plaintes d'un 
homme concernant celle qu'il aime puisqu'elle est incapable de voir le moindre intérêt dans ses collections de fossiles et le moindre sens moral dans ses fouilles archéologiques. Arrive alors un écervelé qui se pavane et fournit l'illustration de la théorie de la sélection sexuelle au locuteur :

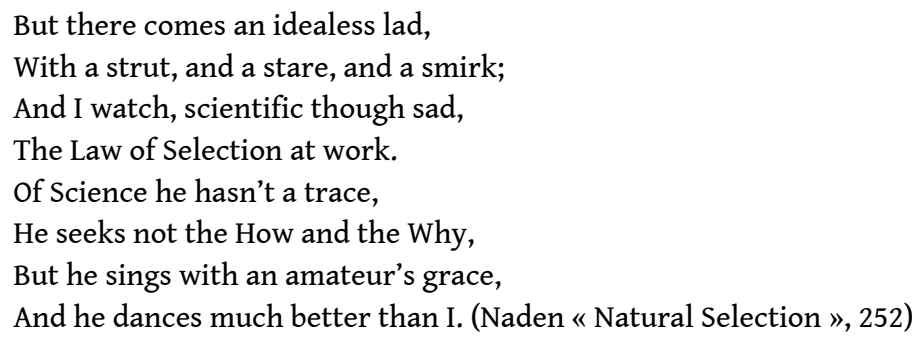

Si la sélection sexuelle s'applique aux oiseaux, pourquoi pas aux humains? La dernière strophe porte le coup fatal aux scientifiques et aux femmes: si Chloé roucoule et montre comment elle est fausse, c'est que Darwin avait raison! Ce poème misogyne qui s'appuie sur la force des caractères sexuels secondaires est aussi une critique de celui qui perçoit le monde comme simplement composé de catégories primaires. Enfin le dernier tableau, « Solomon Redivivus, 1886 », « Salomon Ressuscité », est un monologue dramatique prononcé par le sage biblique Salomon à la reine de Saba qui l'écoute religieusement donner la réponse à une énigme posée hors-champ portant sur l'origine de l'homme. Ils étaient tous deux des amibes heureuses, selon Salomon, jusqu'à ce que la reine de Saba cherche à évoluer de son côté en devenant protozoaire. Leurs chemins se croisent encore alors qu'ils sont sous forme de mollusques puis de poissons, de reptiles puis de mammifères. Si le grand sage et la reine de toute beauté descendent de l'amibe, on peut alors relativiser la valeur de la sagesse et de la beauté, surtout lorsque Salomon prend un ton docte et infantilisant pour expliquer la théorie de l'évolution.

Quatre peintures de mœurs dans quatre poèmes à coloration scientifique et humoristique. Mais malheureusement les scènes décrites tendent plutôt à confirmer par l'exemple les théories darwiniennes. La conception d'une hiérarchie des sexes est à la fois niée et soutenue dans ces quatre volets; la sélection naturelle et le rôle fondamental des caractères sexuels secondaires semblent confirmés chez les humains ; l'évolution poursuit son cours si l'homme n'en est encore qu'à un niveau médiocre d'intelligence. Le constat de Naden est pessimiste, même si elle regarde ses concitoyens avec humanité et peut-être espoir. La critique sociale soutenue par le discours scientifique donne plus de poids au poème qui acquiert une dimension didactique. Cette approche est aussi choisie par May Kendall (1861-1943) dans quelques poèmes évolutionnistes qui rappellent par le trait d'humour et le recours à des situations absurdes et rocambolesques certaines rencontres inopportunes chez Lewis Carroll. Elle ira même jusqu'à mettre en scène des Martiens qui ne comprennent rien à notre planète! Kendall présente des êtres humains dont la vanité, la bêtise ou l'égoïsme ne leur permettent plus de se comparer aux animaux. Elle doute de l'amélioration de l'humain. Dans «The Lay of the Trilobite » (1887), Kendall suggère que l'ignorance du trilobite, c'est-à-dire d'une des formes primitives de vie, est préférable au progrès et à la soi-disant civilisation. Lors d'une promenade en montagne, le narrateur rencontre un trilobite tranquillement occupé à attendre que le temps passe. Il est ému en considérant cet être simple qui est à l'origine de sa vie d'homme. Ce trilobite débute alors sa chanson dans laquelle il rappelle les principes de l'évolution par la sélection naturelle. Il en vient vite à l'époque actuelle qui assiste au développement du génie, 
avec Emmanuel Kant et Robert Browning, sans oublier la revue satirique Punch, mais aussi à l'art de la guerre. Le trilobite quant à lui est un peu stupide : il ne sait pas s'il est vraiment un crustacé ou autre chose, mais il sait qu'il ne se plaint pas, qu'il ne vole pas, qu'il n'écrit pas de poèmes et qu'il peut se contenter d'un verre d'eau salée et d'une assiette de chaux au menu. Le voyageur refuse de s'abaisser à répondre au trilobite, tire sa révérence mais au plus profond de lui, se dit:

I wish our brains were not so good,

I wish our skulls were thicker,

I wish that Evolution could

Have stopped a little quicker;

For oh, it was a happy plight,

of liberty and ease,

To be a simple Trilobite

In the Silurian seas! (Kendall « The Lay of the Trilobite », Blain, 313)

L'évolution n'est pas synonyme de progrès chez Kendall, mais personne ne veut le reconnaître. Et pourtant un ichtyosaure voudrait avoir la chance d'atteindre un état supérieur de l'évolution. C'est dans «A Ballad of the Ichthyosaurus" (1887) qu'il se lamente de ne pas posséder le cerveau supérieur des humains car il ne peut arborer qu'une paire d'yeux gigantesques, certes admirée par les géologues, mais sans comparaison avec le cerveau convoité. Le sien est médiocre, sans commune mesure avec le cerveau de ceux qui peuvent aller à l'université et étudier les ichtyosaures. Il n'aurait même pas réussi à passer le stade de l'examen d'entrée. Personne n'est satisfait de sa condition; l'évolution est un échec, sauf pour ceux qui, en bas de l'échelle, acceptent leur situation avec résignation et une certaine paix intérieure. C'est le cas, dans "The Philanthropist and the Jelly-Fish» (1887), de cette méduse échouée sur la plage qui refuse d'être secourue par le philanthrope, qui, la larme à l'œil, pense au bien qu'il pourrait faire au règne de la méduse s'il pouvait les sauver toutes. Mais la méduse n'a pas de cerveau, pas de "sensorium " pour reprendre le terme qu'elle emprunte aux scientifiques qui la dissèquent. Ainsi flotter dans l'eau salée ou se dessécher sur le sable, le choix lui est bien égal : "I never got depressed and low / Or paralysed by doubt. » (Kendall « The Philanthropist and the Jelly-Fish», 318)

Alors, si les femmes ne sont pas douées pour les sciences, si la poésie est un domaine masculin et s'il est bien connu que les femmes n'ont pas d'humour, faut-il considérer les exemples cités comme des exceptions sans fondement ou bien peut-on voir dans ces poèmes l'expression d'un point de vue éthique véhiculé par des femmes qui se sentent concernées par une approche générale des sciences de l'homme et plus précisément par une réflexion sur la position de la femme au sein de l'humanité et de la production artistique ? Chez Constance Naden, la satire cible surtout les relations amoureuses et les rapports entre les sexes qu'elle analyse au microscope. Chez ses consœurs, c'est l'espèce humaine qui est disséquée et commentée. Écrire la science ne peut, selon les poétesses, être fait sans apporter une dimension éthique qu'elles se chargent d'offrir aux lecteurs. Sans doute prisonnière encore une fois des exigences de l'idéologie essentialiste qui conçoit que la femme transmet sa douceur et son intuition «naturelles », la poétesse scientifique prend place dans l'arène pour rappeler qu'une réflexion sur l'espèce humaine ne peut avoir lieu sans prendre en compte la séparation et la binarité des sexes. Argument à double tranchant, l'utilisation du discours social genré au cœur de la poésie scientifique donne une place à la femme poète mais lui retire en même temps la validité scientifique. La poésie scientifique de femmes reste une poésie de femmes quand les poétesses ne peuvent investir que le champ social et 
psychologique de la science. Plus proche des sciences sociales que des sciences dures, la femme poète parvient toutefois à trouver une voix médiane, celle de l'ironie, qui lui permet de développer un discours psychologique assigné aux femmes tout en adoptant une posture critique, subjective et personnelle. Le discours scientifique sur l'espèce humaine conduit à l'élaboration d'un autre discours qui rejette l'universalité, la norme et la classification et met en évidence une conscience, certes féminine, mais détentrice d'un sens critique, outil de relativisation qui fait la force et la faiblesse du discours féministe. L'ironie et la satire apportent à la rigueur de la science une forme de souplesse et d'adaptabilité qui ne met pas en doute les découvertes révolutionnaires, puisqu'au contraire ces poétesses contribuent à propager et à soutenir les théories darwiniennes. Elles proposent au lecteur d'entendre aussi un autre message atemporel : la perfection n'existe pas. La poétesse refuse la construction du génie scientifique comme celle du génie poétique et introduit un certain degré de proportion inhérent à la poésie de celles qui se positionnent à la fois au cœur et à la périphérie de l'idéologie sexuelle. La poésie témoigne du désir d'infini, mais l'humour rappelle qu'il faut plus modestement relativiser nos ambitions. Mais la relativité est une autre théorie scientifique que l'humour du poète n'a pas non plus épargnée.

\section{BIBLIOGRAPHIE}

Blain, Virginia. Victorian Women Poets: A New Annotated Anthology. Harlow: Longman, 2001.

Blind, Mathilde. The Ascent of Man. London: Chatto and Windus, 1889.

Darwin, Charles. The Descent of Man, and Selection in Relation to Sex. 1871. London: Penguin, 2007.

Leighton, Angela et Reynolds, Barbara. Victorian Women Poets. London: Blackwell, 1995.

\section{RÉSUMÉS}

Lorsque Charles Darwin appliqua ses théories sur la sélection naturelle et sexuelle à l'espèce humaine, dans The Descent of Man, and Selection in Relation to Sex (1871), il alimenta l'ardeur des femmes engagées dans la défense de leurs droits. Certaines poétesses anglaises exprimèrent leur enthousiasme dans une poésie positiviste et progressiste, comme Mathilde Blind, qui voit dans l'évolution des espèces un long chemin vers la perfection. D'autres poétesses, qui mêlent discours scientifique et prise de position autour de la question du genre, dénoncent l'essentialisme construit par le Darwinisme. Louisa Bevington, A. Mary F. Robinson, Constance Naden et May Kendall utilisent les résultats des théories sur la sélection naturelle et sexuelle afin de mettre en évidence, avec humour et ironie, les relations asymétriques entre les sexes et le ridicule de l'être humain lorsqu'il a conscience de sa supériorité.

When Charles Darwin applied his theories on natural and sexual selection to the human race in The Descent of Man, and Selection in Relation to Sex (1871), he fostered the fervour of the women committed to the defence of their rights. Some women poets expressed their enthusiasm in their positivist and progressive poetry, such as Mathilde Blind, who considered the evolution of 
species as a long path towards perfection. Other women poets, who used scientific discourse to challenge the woman question, denounced the essentialist vision springing from Darwinism. Louisa Bevington, A. Mary F. Robinson, Constance Naden et May Kendall used the theories of natural and sexual selection in order to highlight with humour or irony the asymmetrical relations between the sexes and to show how ridiculous human beings can be when they are fully aware of their superiority.

\section{INDEX}

Keywords : poetry, science, evolution, natural selection, gender, essentialism, humour Mots-clés : poésie, évolution, science, sélection naturelle, genre, essentialisme, humour

\section{AUTEURS}

\section{FABIENNE MOINE}

Maître de conférences

Université Paris Ouest Nanterre la Défense

fmoine@u-paris10.fr 